Bayero Journal of Pure and Applied Sciences, 11(1): 45 - 49

ISSN 2006 - 6996

\title{
GAUSSIAN BEAM DIVERGENCE USING PARAXIAL APPROXIMATION
}

\author{
Busari, ${ }^{1}$ T.A., Kashimbila, ${ }^{2}$ M.M., Busari, ${ }^{3}$ K.A., Afolabi, ${ }^{4}$ M.A. and Adamu, I.D. \\ ${ }^{1}$ Nigerian Tulip International college, Kano, Nigeria \\ ${ }^{2,5}$ Department of Physics, Bayero University, Kano, Nigeria \\ ${ }^{3}$ Department of Physics, Federal University, Gashua Yobe State, Nigeria \\ ${ }^{4}$ The light College, Kano, Nigeria \\ Corresponding author: jeliltaiwo@gmail.com, 08141548015, 07053548164, 08176102707
}

\begin{abstract}
Gaussian beam intensity follows a normal distribution curve in free space. As it propagates, the divergence and the width increase with distance. Using paraxial approximation, the effect of the distance on the intensity, width, radius of curvature and divergence was investigated. Matlab software was used for characteristics parameters calculation. The divergence angle range $\left(26.67^{\circ} \leq \theta \leq 35.85^{\circ}\right)$ was obtained at a distance between $3 \mathrm{~mm}$ and $4 \mathrm{~mm}$, and compared with the divergence angle range $\left(43^{\circ} \leq \theta \leq 53^{\circ}\right)$ obtained experimentally for the distance between $3 \mathrm{~mm}$ and $4.5 \mathrm{~mm}$. The result obtained in this work indicates smaller angles of divergence that can produce a better beam quality and intensity. Finally, this will serve as a great importance for application such as pointing, free space optical communication etc.

Keyword: Gaussian beam, Paraxial approximation, Beam divergence, Normal distribution curve.
\end{abstract}

INTRODUCTION

Interests in high-data-rate free-space optical (FSO) laser communication systems have grown significantly in recent years because of some advantages offered by FSO systems over radio frequency (RF) systems. FSO systems include three basic subsystems: transmitter, channel and receiver (Andrews and Phillips, 1998). Transmitter and receiver include some optical elements to reduce signal-to-noise ratio (SNR) by optimizing divergence and focusing parameters in transmitter and receiver, respectively.

Therefore, the propagation of laser beams through complicated optical systems is a subject of theoretical and practical interests (Zhao et al., 2003) and some exact solutions of the wave equations were obtained for some certain initial conditions and symmetries ( $\mathrm{Yu}$ et al., 2002). Within the frame work of the paraxial approximation, it is well known that the beam propagation through a paraxial $A B C D$ system is characterized by the Collins formula. In studying the laser beam propagation a great effort has been devoted to find approximate analytical propagation equations, which provide advantages not only of saving the computing time, but also of giving the intuitive physical insight into the beam propagation properties.

Recently, free-space optical (FSO) systems are competitively being installed as broad band access communication links. Depending on the application, a laser beam with a different shape may have favorable characteristics over a fundamental mode Gaussian beam. In particular, there is current interest in finding out whether beam shaping can be used as an effective counter measure to extend the propagating range beyond several hundred meters. In this sense, FSO system performance as related to different types of laser incidence has been analyzed.

Before introducing the details of atmospheric characteristics in FSO links, it is of interest to understand the free-space propagation characteristics of the special higher order annular Gaussian (HOAG) laser beams obtained by subtracting a smaller size (secondary) Hermite-Gaussian beam from a larger size (primary) Hermite-Gaussian beam, with different size. Hermite-sinusoidal-Gaussian HSG (Baykal, 2004) and a special case of HSG laser beams in turbulent FSO links (Tanyer, 2004) were also introduced. Since the ordinary Hermite-Gaussian beam solutions are already known to form a complete set of solutions of the paraxial wave equation and since HOAG beams are expressible as a linear combination of two Hermite-Gaussian beams, these beams also forms a complete set. It is also useful to check variation of the beam shape in different beam shaping media. For example, a report by (Hedayati et al., 2010) explains characterizing the divergence properties of the laser diode beams propagation through collimator and aperture optical system. Nonlocal nonlinear media (NNM) have attracted a lot of attention in the past decade. 
Many novel solutions in NNM have been presented which have no counterparts in traditional local nonlinear media, and exhibit many novel phenomena (Horikis and Frantzeskakis, 2016). In recent years, the shape-variant optical beams in NNM have also received intensive attention (Zhang, 2016).

Also, some unique characteristics are revealed, such as self-induced mode transformation (Izdebskaya et al., 2013) power variationinduced three-dimensional non-uniform scaling (Lu et al., 2013). It is well known that Gaussian beams represent a realistic model for the description of the field of laser modes of many laser systems. However, Gaussian beam solutions can easily be obtained only in the paraxial approximation of the wave equation or of the Maxwell equations.

This work is founded on the Maxwell equations, from which the Helmholtz equation is obtained. The Helmholtz equation was now solved analytically using the paraxial approximation to obtain the paraxial wave equation (PWE). From the solution of (PWE), Gaussian beam width, radius of curvature, divergence and intensity equations were obtained and simulated.

\section{Background Theory}

The starting points of this work are the Maxwell's Equations below (Guenther, 1990):

$\vec{\nabla} \times \overrightarrow{\mathrm{E}}=-\frac{\partial \overrightarrow{\mathrm{B}}}{\partial \mathrm{t}}$

$\vec{\nabla} \times \overrightarrow{\mathrm{B}}=\mu_{0}\left(\overrightarrow{\mathrm{J}}+\varepsilon_{0} \frac{\partial \overrightarrow{\mathrm{E}}}{\partial \mathrm{t}}\right)$

$\vec{\nabla} \cdot \overrightarrow{\mathrm{E}}=\frac{\rho}{\varepsilon_{0}}$

$\vec{\nabla} \cdot \overrightarrow{\mathrm{B}}=0$

where $\mathrm{J}=$ Current Density, $\sigma=$ Electrical Conductivity, $\mathrm{E}=$ Electric field,

$B=$ Magnetic field, $\rho=$ Charge Density, $\mu_{0}=$ permeability in free space, $\varepsilon_{0}=$ permitivity in free space

HELMHOLTZ EQUATION

For free space, the differential equation that must be satisfied to determine the spatial behavior of a wave is the Helmholtz equation. This equation is given as follows (Guenther, 1990):

$\nabla^{2} \overrightarrow{\mathrm{E}}+\frac{\omega^{2}}{\mathrm{c}^{2}} \overrightarrow{\mathrm{E}}=0$

$\mathrm{k}=\frac{\omega}{\mathrm{c}}$

$\nabla^{2} \overrightarrow{\mathrm{E}}+\mathrm{k}_{0}^{2} \overrightarrow{\mathrm{E}}=0$

angular frequency where $\mathrm{k}_{0}=$ wave number, $\mathrm{c}=$ speed of light in vacuum, $\omega=$

\section{Paraxial Approximation}

Gaussian beams are usually considered in situations where the beam divergence is relatively small, so that the so called paraxial approximation can be applied. This approximation assumes that the propagation direction of light is very close to the $z$ axis and that the propagation distance along this axis is much greater than the transverse spreading of the wave.

Considering a wave equation in the Helmholtz expression (Guenther, 1990):

$\frac{\partial^{2} \mathrm{E}}{\partial \mathrm{x}^{2}}+\frac{\partial^{2} \mathrm{E}}{\partial \mathrm{y}^{2}}+\frac{\partial^{2} \mathrm{E}}{\partial \mathrm{z}^{2}}+\mathrm{k}^{2} \mathrm{E}=0$

Where $E(x, y, z)$ represents the complex amplitude of a scalar and monochromatic optical field that is propagated in free space.

Assuming a scalar wave of the form which propagates nearly parallel to the $z$ axis is expressed as:
$E(r)=\Psi(x, y, z) e^{-i k z}$

Substituting equation (9) into the Helmholtz equation, produce the scalar paraxial wave equation below, which must be solved (Guenther, 1990).

$$
\begin{aligned}
\left(\frac{\partial^{2} \Psi}{\partial \mathrm{x}^{2}}+\frac{\partial^{2} \Psi}{\partial \mathrm{y}^{2}}+\frac{\partial^{2} \Psi}{\partial \mathrm{z}^{2}}\right) \mathrm{e}^{-\mathrm{ikz}}+\mathrm{k}^{2} \Psi \mathrm{e}^{-\mathrm{ikz}} \\
-2 \mathrm{ik} \frac{\partial \Psi}{\partial \mathrm{z}} \mathrm{e}^{-\mathrm{ikz}}-\mathrm{k}^{2} \Psi \mathrm{e}^{-\mathrm{ikz}} \\
=0
\end{aligned}
$$

Using the paraxial approximation from equation (10), we have:

$\frac{\partial^{2} \Psi}{\partial \mathrm{x}^{2}}+\frac{\partial^{2} \Psi}{\partial \mathrm{y}^{2}}-2 \mathrm{ik} \frac{\partial \Psi}{\partial \mathrm{z}}$

$=0$ 
The solution of (11) gives:

$\Psi=\mathrm{e}^{-\mathrm{iQ}(\mathrm{z})\left(\mathrm{x}^{2}+\mathrm{y}^{2}\right)} \mathrm{e}^{-\mathrm{iP}(\mathrm{z})}$

$\mathrm{Q}(\mathrm{z})$ is a complex variable associated with the reciprocal of the gaussian width,

$\mathrm{P}(\mathrm{z})$ contain information on the phase of the wave

Finding the derivatives of (12) and substituting into (11), we obtain

$$
\Psi=\mathrm{e}^{-\mathrm{i}\left[-\mathrm{i} \ln \left(1+\frac{\mathrm{z}}{\mathrm{q}_{0}}\right)+\frac{\mathrm{k}}{2\left(\mathrm{q}_{0}+\mathrm{z}\right)}\left(\mathrm{x}^{2}+\mathrm{y}^{2}\right)\right]}
$$

From equation (13) we have

$$
\Psi=\frac{1}{\sqrt{1+\left(\frac{z}{q_{0}}\right)^{2}}} e^{\mathrm{a}} \mathrm{e}^{\mathrm{b}}
$$

If $a=\left[-\frac{\mathrm{kq}_{0}\left(\mathrm{x}^{2}+\mathrm{y}^{2}\right)}{2\left(\mathrm{z}^{2}+\mathbf{q}_{0}^{2}\right)}\right]$ and $\mathrm{b}=\left[\mathrm{i} \tan ^{-1}\left(\frac{\mathrm{z}}{\mathbf{q}_{0}}\right)-\frac{\mathrm{ikz}\left(\mathrm{x}^{2}+\mathrm{y}^{2}\right)}{2\left(\mathrm{z}^{2}+\mathbf{q}_{0}^{2}\right)}\right]$, then

At $z=0$, equation $(14)$ reduces to

$$
\Psi_{0}=\mathrm{e}^{\left[-\frac{\mathrm{k}\left(\mathrm{x}^{2}+\mathrm{y}^{2}\right)}{2 \mathbf{q}_{0}}\right]}
$$

Equation (15) is a Gaussian function which can be compared with a Gaussian spatial amplitude distribution given by:

$$
\mathrm{E}=\mathrm{E}_{0} \mathrm{e}^{\left[-\left(\frac{\mathrm{x}^{2}+\mathrm{y}^{2}}{\mathrm{w}^{2}}\right)\right]}
$$

where, $w_{0}$ is the beam waist, $\Psi_{\text {is }}$ a wave whose amplitude distribution is a Gaussian, and $\mathrm{q}_{0}$ is called confocal parameter which is given by:

$\mathbf{q}_{0}=\frac{\pi w_{0}^{2}}{\lambda}$

$$
\mathrm{q}_{0}=\mathrm{i} \mathbf{q}_{0}
$$

Using the definition of $q_{0}$ given in above, we can write (14) as:

$$
\begin{aligned}
& \Psi=\frac{1}{\sqrt{1+\left(\frac{\lambda z}{\pi w_{0}^{2}}\right)^{2}}} e^{c} e^{d} \\
& \text { Where } c=\frac{\left(x^{2}+y^{2}\right)}{w_{0}^{2}\left[1+\left(\frac{\lambda z}{\pi w_{0}^{2}}\right)^{2}\right]} \text { and } d=i \tan ^{-1}\left(\frac{\lambda z}{\pi w_{0}^{2}}\right)-\frac{i \pi\left(x^{2}+y^{2}\right)}{\lambda z\left[1+\left(\frac{\pi w_{0}^{2}}{\lambda z}\right)^{2}\right]}
\end{aligned}
$$

From the real part of equation (17), the following equations are obtained:

$$
\begin{aligned}
& w^{2}(z)=\mathrm{w}_{0}^{2}\left[1+\left(\frac{\lambda \mathrm{z}}{\pi \mathrm{w}_{0}^{2}}\right)^{2}\right] \\
& \mathrm{E}=\mathrm{E}_{0} \mathrm{e}^{\left[-\left(\frac{\mathrm{x}^{2}+\mathrm{y}^{2}}{\mathrm{w}^{2}}\right)\right]}
\end{aligned}
$$

While from the imaginary part the following equations are obtained:

$$
\begin{aligned}
& R(z)=\mathrm{z}\left[1+\left(\frac{\pi \mathrm{w}_{0}^{2}}{\lambda \mathrm{z}}\right)^{2}\right] \\
& \theta_{d} \cong \tan \theta_{d}=\frac{w}{z}=\frac{\lambda}{\pi w_{0}}
\end{aligned}
$$

Equation (18) and (19) are Gaussian beam width and intensity respectively. While equation (20) and (21) are radius of curvature and divergence respectively.

\section{MATERIALS AND METHODS}

Paraxial approximation was used to obtain equation (18), (19), (20) and (21), after which• they were computationally simulated in the Matlab environment. The procedures for the simulation consist of the following steps:opening the MATLAB environment, new script, save and command window.

- Firstly, we opened the MATLAB.

- In the MATLAB environment, we clicked on new script to open the editor, after which a script was written to simulate equation (18), (19), (20) and (21).

In the script of equation (18) and (20), we set $\lambda$ $>760 \mathrm{~nm}$ which has to be within the infrared region, $\mathrm{z}=0$ to $12 \mathrm{~mm}, w_{0}=4.15 \mathrm{~mm}$.

In the script of equation (21), we also set $z=0$ to $12 \mathrm{~mm}$ and obtained the divergence since the beam width at different propagation distance is known. 
- In the script of equation (19), we set $\mathrm{E}_{0}$ as a unit amplitude, $\mathrm{w}=4.15 \mathrm{~mm}, 6.9811 \mathrm{~mm}$ and $11.09701 \mathrm{~mm}$. Thereafter $y$ is fixed as 0 and $x$ varied from $-12 \mathrm{~mm}$ to $+12 \mathrm{~mm}$ to obtain the intensity.

- The following are some of the commands used in the script: function name e.g. function [p1, p2, p3] = Gauss [y], grid on, hold on, plot, $x$ label and y label, title, legend, end etc.

- To save the script, the save was clicked.

- On the command window, the function names were entered to run the script.

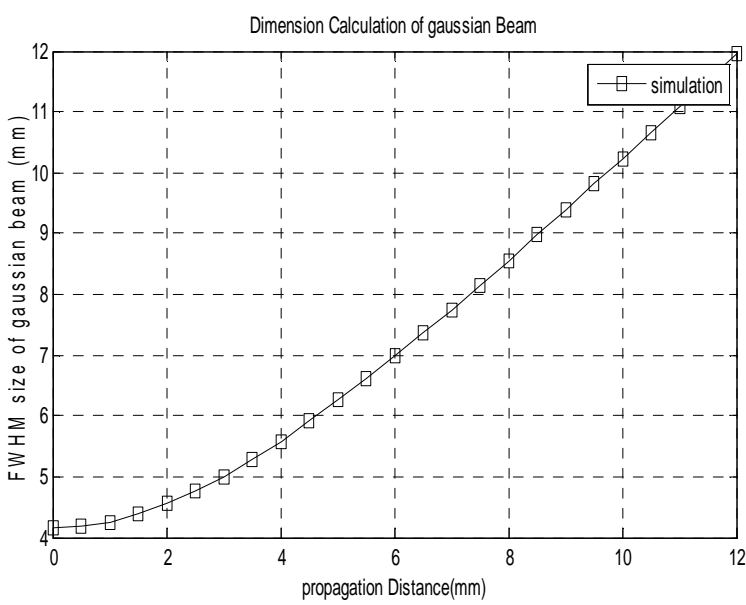

Figure 1: Gaussian beam size as a function of propagation distance.
Thereafter, Different values of the beam width, divergence, radius of curvature and intensity were obtained as a function of propagation distance.

Finally, we obtained the graph of the beam width, divergence, radius of curvature and intensity against propagation distance.

\section{RESULTS AND DISCUSSION}

From computational analysis through simulation, the following results were obtained as given in the figures (1, 2, 3 and 4) below:

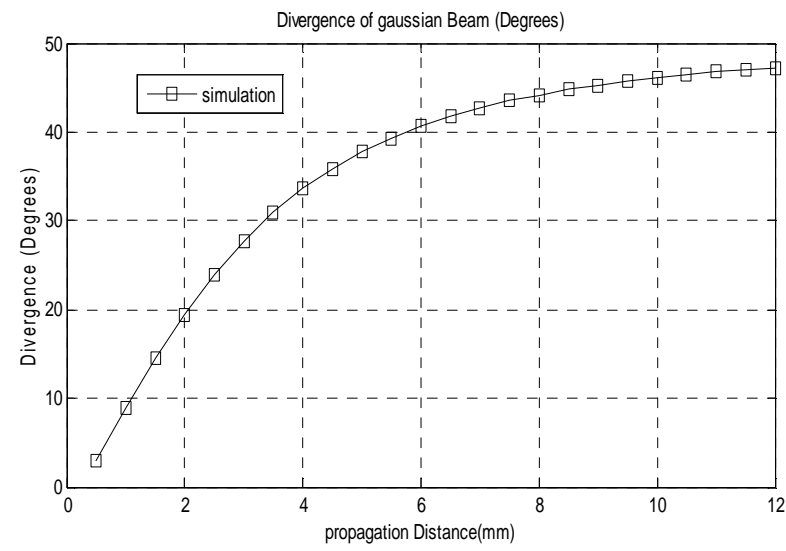

Figure 2: Gaussian beam divergence as a function of propagation distance.

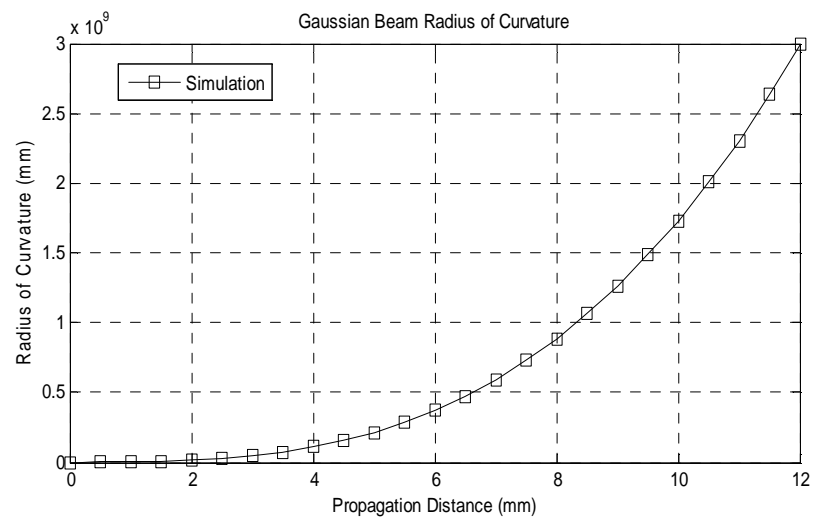

Figure 3: Gaussian beam radius of curvature as a function of propagation distance.

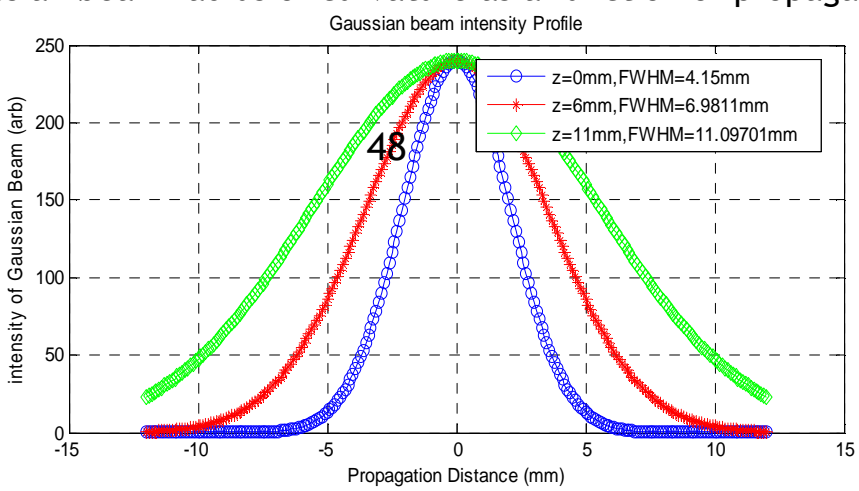

Figure 4: Intensity Profile of the Gaussian Beam as a function of propagation Distances 
Special Conference Edition, November, 2018

In Figure 1, the beam dimension as a function of propagation is presented. By using the fitting options and the governing equation to the points in the MATLAB program, the FWHM size of the beam were obtained. The result of this calculation shows some important points.

First point shows that the calculated beam size values were increasing by increasing the propagation distance. As it can also be seen in Figure 1, variation of the beam size shows a linear dependence on the distance.

Figure 2 presents the divergence of the Gaussian beam as a function of the propagation distance. The governing equation indicates divergence angle range of $\left(27.67^{\circ} \leq \theta \leq\right.$ $35.85^{\circ}$ ), at a distance between $3 \mathrm{~mm}$ and $4.5 \mathrm{~mm}$. It can be noted that as the propagation distance increases, the divergence also increases, due to increase in the beam size.

Figure 3 represents the Gaussian beam radius of curvature as a function of the propagation distance. From $0 \mathrm{~mm}$ to $12 \mathrm{~mm}$ propagation distance, the Gaussian beam radius of curvature increased from $0 \mathrm{~mm}$ to2.99 $\times$ $10^{9} \mathrm{~mm}$. Therefore, it was noted that as the propagation distance tends to infinity, the radius of curvature shows a linear variation with the distance, because in the far field, the wave front radius of curvature is planar.

Figure 4 represents the beam intensity as a function of distance values. By using the fitting

\section{REFRENCES}

Andrews, L.C., and R.L, Phillips, (1998). Laser beam propagation through random media. Bellingham, Washington USA: SPIE optical Engineering press, A publication of SPIE, the international Society for optical Engineering.

Baykal, Y. (2004). Correlation and structure functions of Hermite - sinusoidalGaussian, 21(7), 1290-1299.

Guenther, R.D. (1990). Modern Optics, John Willey, pg 342.

Hedayati, M. R., F. D. Kashani, M. M. Eftekhari, and M. R. Mahzoun, (2010). Characterizing the divergence properties of the laser diode beams propagation through collimator and aperture $A B C D$ optical system. Optics and Laser Technology, 42(8), 1269-1275. http://doi.org/10.1016/j.optlastec.2010. 03.021

Horikis, T.P. and D.J. Frantzeskakis, (2016). Ring dark and antidark solitons in nonlocal media Optics Letter; 41:583.

Izdebskaya, Y.V, A.S. Desyatnikov and Y.S. Kivshar, (2013). Self-Induced Mode options in the MATLAB program and the governing equation to the points, the beam intensities and the intensity profiles at different propagation distances were obtained. Also, Figure 4 shows the intensity profiles for the $z$ values of 0,6 and $11 \mathrm{~mm}$. the intensity of the beam is plotted as a function of distances from $-12 \mathrm{~mm}$ to $+12 \mathrm{~mm}$. it is noted that the intensity profile follows a Gaussian distribution in the free space. As shown in figure 4.2, the FWHM is about $4.15 \mathrm{~mm}$ at $\mathrm{z}=0 \mathrm{~mm}$, while it is about $6.9811 \mathrm{~mm}$ and $11.09701 \mathrm{~mm}$ for $z=6 \mathrm{~mm}$ and $\mathrm{z}=11 \mathrm{~mm}$ respectively.

However, from Figure 4, considering the change in the propagation distance, there is notable difference between band widths. Thus, FWHM is increased by increasing the propagation distance due to beam divergence.

\section{CONCLUSION}

It was shown in this work that, a long propagation distance in the free space leads to low intensity, large beam spot size, radius of curvature, divergence and vice versa. Considering the computed result, Also, it was found that beam with low divergence has better beam quality and intensity which can be important for applications such as pointing, free space optical communications and optical pumping of lasers by high power diode arrays.

Transformation in Nonlocal Nonlinear Media Physical Review Letters.; 111:123902.

Lu, D., Q. Zhan, Q. Duan, and W. Hu, (2013) Power-variation-induced threedimensional nonuniform scaling of beams in strongly nonlocal nonlinear media Physics Review A; 87:023815.

Tanyer, H. (2004). Analysis of reciprocity of cos-Gaussian and cosh- Gaussian laser beams in a turbulent atmosphere, 12(14), 1187-1192.

Yu, S., H. Guo, X. Fu, and W. Hu, (2002). Propagation properties of elegant Hermite - cosh-Gaussian laser beams. Optical Communication, 204(April), 5966.

Zhang, X. (2016). A novel splice donor site mutation in EPHA2 caused congenital cataract in a Chinese family. Optical Communication, 367:364.

Zhao, G., X. Ji, and B. Lu, (2003). Approximate analytical propagation equations of Gaussian beams through hard-aperture optics, 6(6), 241-245. 